\title{
A auto-estima de indivíduos que foram submetidos à cirurgia de revascularização do miocárdio
}

THE SELF-ESTEEM OF INDIVIDUAL WHO HAVE BEEN SUBMITTED TO CORONARY ARTERY BYPASS GRAFT SURGERY

LA AUTOESTIMA DE INDIVIDUOS QUE FUERON SOMETIDOS A CIRURGÍA DE REVASCULARIZACIÓN DEL MIOCARDIO

Tatiana Victorelli Pires Vargas ${ }^{1}$, Rosana Aparecida Spadoti Dantas ${ }^{2}$, Cristiane Franca Lisboa Gois ${ }^{3}$

1 Bolsista de Iniciação Científica (PIBIC/USP/ $\mathrm{CNPq})$. Aluna de graduação da Escola de Enfermagem de Ribeirão Preto -Universidade de São Paulo (EERP-USP)

2 Professora-Doutora do Departamento de Enfermagem Geral e Especializada - EERP-USP. rsdantas@eerp.usp.br

3 Enfermeira do Hospital das Clínicas da Faculdade de Medicina de Ribeirão Preto (FMRP-USP) cristianeflg@hotmail.com

\begin{abstract}
RESUMO
Estudo descritivo e correlacional com objetivos de medir a autoestima de indivíduos submetidos à cirurgia de revascularização do miocárdio e correlacionar a autoestima com variáveis sóciodemográficas e clínicas. Uma amostra de 97 pacientes ambulatoriais foi entrevistada. Os dados foram analisados através de estatística descritiva, teste de Correlação de Pearson, testes MannWhitney e Kruskal Wallis. A confiabilidade da escala foi verificada (alfa de Cronbach de 0,79 ) e a média encontrada para auto-estima foi 32,22 (intervalo possível de 10 a 40). Encontramos correlação, estatisticamente significante, apenas para autoestima e sexo. Concluímos que os participantes apresentavam elevada auto-estima a qual não estava correlacionada com outras variáveis.
\end{abstract}

\section{DESCRITORES}

Auto-imagem.

Qualidade de vida.

Revascularização miocárdica.

\section{ABSTRACT}

This is descriptive and correlational study that measured the self-esteem of individuals submitted to coronary artery bypass graft surgery and correlated the self-esteem with socio-demographic and clinical variables. A sample of 97 outpatients was interviewed. Data were analyzed by means of descriptive statistics, Pearson's Correlation test, Mann-Whitney and Kruskal Wallis tests. The reliability of the scale was verified (Cronbach alfa was 0.79) and the average for self-esteem was found to be 32.22 (possible range from 10 to 40 ). Statistically significant correlation was found only between self-esteem and gender. The conclusion is that the participants had a high level of self-esteem, which was not correlated to other variables.

\section{KEY WORDS}

Self-image.

Quality of life.

Miocardial revascularization.

\section{RESUMEN}

Estudio descriptivo y correlacional realizado con los objetivos de medir la autoestima de individuos sometidos a cirugía de revascularización del miocardio y de correlacionar la autoestima con variables sociodemográficas y clínicas. Fue entrevistada una muestra de 97 pacientes de consulta externa. Los datos fueron analizados por estadística descriptiva, test de Correlación de Pearson, tests Mann-Whitney y Kruskal Wallis. Se verificó la confiabilidad de la escala alfa de Cronbach de 0,79 y la media encontrada para autoestima fue de 32.22 (intervalo posible de 10 a 40). Encontramos correlación, estadísticamente significativa, apenas para autoestima y sexo. Concluimos que los participantes presentaban elevada autoestima, la cual no estaba correlacionada con otras variables.

\section{DESCRIPTORES}

Autoimagem.

Calidad de vida.

Revascularización miocárdica. 


\section{INTRODUÇÃO}

A auto-estima de indivíduos com doenças cardíacas tem sido estudada com relação a diversos aspectos, dentre eles a qualidade de vida desses indivíduos. Elevada auto-estima tem sido associada a baixos níveis de depressão e tensão ${ }^{(1)}$, favorável recuperação social e física ${ }^{(2)}$ e elevada qualidade de vida após eventos cardíacos ${ }^{(3)}$ e após revascularização do miocárdio ${ }^{(4)}$.

A cirurgia de revascularização do miocárdio (RVM) é um dos procedimentos cirúrgicos mais comumente realizados. Como outras formas de tratamento da doença arterial coronariana (DAC), esta cirurgia não é curativa e deve ser acompanhada por outras medidas terapêuticas como uso de medicamentos e mudanças no estilo de vida, visando o controle da progressão da doença coronariana. A DAC é uma doença crônica e pode influenciar na qualidade de vida de seus portadores ${ }^{(3-4)}$. Alguns autores têm desenvolvido modelos teóricos que buscam explicar as variações existentes na qualidade de vida de doentes cardíacos. Em estudo com pacientes após ataque cardíaco a inclusão da variável auto-estima, no modelo proposto pelos autores, foi responsável por um aumento de $25 \%$ na variação da qualidade de vida, ou seja, contribuiu de forma positiva e significante para aumentá-la ${ }^{(3)}$. Entre pacientes submetidos à revascularização cirúrgica do miocárdio a variação, no modelo explicativo de qualidade de vida, foi de $5 \%$ com a inclusão da variável auto-estima ${ }^{(4)}$.

\section{REFERENCIAL TEÓRICO}

Definiu-se auto-estima como uma atitude positiva ou negativa para com um objeto particular chamado de self. No entanto, auto-estima tem duas diferentes conotações. Uma conotação de alta auto-estima é aquela cuja pessoa pensa que ela é "muito boa"; uma conotação muito diferente daquela na qual o indivíduo acha-se "bom o suficiente". É possível que um indivíduo, embora considerando-se superior aos demais, possa sentirse inadequado em termos de certos padrões que estabeleceu para si mesmo. Alta auto-estima, como refletida na escala de Rosenberg ${ }^{(5)}$, expressa o sentimento que o indivíduo tem em achar-se "bom o suficiente". O indivíduo respeita a si mesmo, considera-se capaz sem, necessariamente, sentir-se superior às outras pessoas. Definitivamente, ele não se considera pior que os demais, embora reconheça suas limitações e expectativas para crescer e se aperfeiçoar. Por outro lado, baixa auto-estima implica em auto-rejeição, insatisfa- ção consigo mesmo e desprezo por sua própria pessoa. Sua auto-imagem é ruim, e ele deseja esconder-se. O grau de aceitação ou de rejeição do self, é um fenômeno de aprendizado que envolve todo o processo da vida do indivíduo ${ }^{(5)}$.

Então, podemos nos questionar o por que da importância de nós, enfermeiros, bem como de outros profissionais da área de saúde, nos preocuparmos com a auto-estima dos indivíduos sob os nossos cuidados. Ao nosso ver, principalmente nas áreas das doenças crônicas, como a doença arterial coronariana, os doentes e seus familiares terão que compreender o significado da enfermidade, aprender a conviver com ela e saber quais serão os ganhos/perdas diante das suas decisões. Neste contexto, vários estudiosos têm procurado definir e operacionalizar conceitos como auto-estima, auto-eficácia, enfrentamento (coping) e apoio social.

Há controvérsias sobre as definições dos constructos: auto-estima e auto-eficácia. Há evidências empíricas indicando uma forte inter-relação entre os mesmos ${ }^{(6)}$. Auto-eficácia tem sido considerado um conceito novo e, até mesmo, controverso ao conceito de auto-estima. Auto-eficácia tem sido referida como a(s) capacidade(s) do indivíduo para realizar/conduzir tarefas com confiança e sucesso $^{(6-7)}$.

No caso dos indivíduos com DAC, para promover a reabilitação após a cirurgia de revascularização do miocárdio, eles precisam, muitas vezes, mudar comportamentos (deixar de fumar, controlar o estresse, mudar a dieta alimentar) e assumir novas tarefas (praticar exercícios físicos e usar medicamentos). Auto-estima e auto-eficácia seriam fatores que influenciariam as mudanças de comportamentos. Um indivíduo com baixa autoeficácia e auto-estima, provavelmente, teria uma menor expectativa com relação ao seu desempenho em promover e manter a mudança necessária para a sua recuperação.

A auto-estima dos indivíduos pode ser influenciada pelas características sociais como sexo, idade e estado civil, bem como pelas doenças que os acometem ${ }^{(8-9)}$. Assim, investigar se existe uma relação entre essas variáveis e auto-estima de pacientes revascularizados poderá auxiliar o enfermeiro a planejar de forma mais consistente à assistência a esses indivíduos, mostrando aqueles pacientes que são mais vulneráveis durante o processo de reabilitação.

Nesse estudo, optamos por adotar como referencial teórico o conceito de auto-estima pro-
A auto-estima de indivíduos que

foram submetidos à cirurgia de revascularização do miocárdio 
Tatiana Victorelli P. Vargas Rosana Ap. S. Dantas Cristiane Franca L. Gois posto por Rosenberg ${ }^{(5)}$ visando conhecer e medir a auto-estima de indivíduos com doença arterial coronariana e que foram submetidos à cirurgia de revascularização do miocárdio.

\section{OBJETIVOS}

Diante do exposto, este estudo teve como objetivos:

- mensurar a auto-estima de pacientes após cirurgia de revascularização do miocárdio usando a Escala de Auto-Estima de Rosenberg ${ }^{(5)}$, em sua versão traduzida e adaptada para a língua portuguesa $^{(10)}$;

- correlacionar a variável auto-estima com variáveis sócio-demográficas e clínicas dos pacientes.

\section{METODOLOGIA}

\section{Design, amostragem e local do estudo}

Um estudo do tipo descritivo e correlacional foi utilizado para mensurar e correlacionar a autoestima de pacientes revascularizados com outras variáveis sócio-demográficas e clínicas. A amostragem deste estudo constou de 97 pacientes que foram submetidos à cirurgia de revascularização do miocárdio, entre 1996 e 2001 , e estavam em seguimento ambulatorial. Os participantes do estudo eram considerados inelegíveis se: tivessem sido submetidos à cirurgia de RVM com concomitante realização de outra cirurgia torácica (como correção ou troca de válvulas); possuíssem doenças psiquiátricas, como demência e esquizofrenia ou não concordassem em participar da pesquisa. $\mathrm{O}$ estudo foi realizado no Hospital das Clínicas de Ribeirão Preto da Universidade de São Paulo, junto à Divisão de Cardiologia.

\section{Coleta dos dados}

O projeto de pesquisa foi aprovado pelo Comitê de Ética do Hospital das Clínicas de Ribeirão Preto da Faculdade de Medicina da Universidade de São Paulo. Os potenciais participantes foram convidados a participarem da pesquisa, cujos objetivos foram apresentados e, com a concordância dos mesmos, o Termo de Consentimento Livre e Esclarecido foi assinado pelo participante e pesquisador. Os dados foram coletados através de entrevistas individuais com os pacientes, antes do atendimento médico, no ambulatório de Cardiologia.
Quando o participante não podia preencher o instrumento por dificuldades físicas (como déficit de visão) ou cognitivas (não saber ler e/ ou escrever), ele era entrevistado por um dos pesquisadores.

\section{Descrição dos instrumentos de coleta dos dados}

Foi utilizado um questionário contendo itens para a caracterização sócio-demográfica (sexo, idade, estado civil, desempenho de atividades remuneradas) e clínicas (número de enxertos coronarianos e interferência da cirurgia na qualidade de vida) dos participantes. A varíavel dependente, auto-estima, foi medida através da Escala de Auto-Estima de Rosenberg(5), em sua versão traduzida e adaptada para o português ${ }^{(10)}$. Trata-se de uma escala de 4 pontos do tipo Likert $(1=$ concordo fortemente, $2=$ concordo, 3=discordo, 4=discordo fortemente), contendo 10 itens. Desse total de itens, 5 avaliam sentimentos positivos do indivíduo sobre si mesmo (De modo geral, estou satisfeito comigo mesmo; Eu sinto que tenho um tanto de boas qualidades; Eu sou capaz de fazer coisas tão bem quanto a maioria das outras pessoas, desde que me ensinadas; Eu sinto que sou uma pessoa de valor, pelo menos num plano igual às outras pessoas; Eu tenho uma atitude positiva em relação a mim mesmo) e 5 avaliam sentimentos negativos (As vezes, eu acho que não sirvo para nada; Não sinto satisfação nas coisas que realizei. Eu sinto que não tenho muito do que me orgulhar; As vezes, eu realmente me sinto inútil, incapaz de fazer as coisas; Eu gostaria de ter mais respeito por mim mesmo; Quase sempre eu estou inclinado a achar que sou um fracassado). Para a pontuação das respostas, os 5 itens que expressam sentimentos positivos têm valores invertidos e, então, soma-se esses valores aos dos outros 5 itens, totalizando um valor único para a escala, correspondente às respostas dos 10 itens. $\mathrm{O}$ intervalo possível dessa escala é de 10 (10 itens multiplicados por valor 1) a 40 (10 itens multiplicados por valor 4). Alta auto-estima, como refletida na escala de Rosenberg, expressa o sentimento que o indivíduo tem em achar-se "bom o suficiente". O indivíduo respeita a si mesmo, considera-se capaz sem, necessariamente, sentir-se superior às outras pessoas. Alta pontuação indica elevada auto-estima. Definitivamente, ele não se considera pior que os demais, embora reconheça suas limitações e expectativas para crescer e se aperfeiçoar. Por outro lado, baixa auto-estima implica em auto-rejeição, insatisfação consigo mesmo e desprezo por sua própria pessoa. 


\section{Análise dos dados}

Os dados foram analisados usando o programa de software Statistical Package for Social Science (SPSS) versão 10.0, para cálculo das análises descritivas e de correlação. Coeficientes correlacionais de Pearson foram calculados entre auto-estima e as variáveis: idade e tempo de revascularização do miocárdio. Para analisar as diferenças, encontradas na variável auto-estima, no que se refere às variáveis categóricas utilizamos os testes não paramétricos de Mann-Whitney (sexo, estado civil e ocupação) e o de Kruskal Wallis (percepção da qualidade de vida e número de enxertos). O nível de significância adotado foi de 0,05 .

\section{RESULTADOS}

Uma amostra de conveniência, constituída por 97 pacientes ambulatoriais, foi entrevistada. Entre os entrevistados, $76(78,6 \%)$ optaram para que o preenchimento do instrumento de coleta fosse feito pela pesquisadora alegando problemas cognitivos ou físicos. A caracterização sóciodemográfica e clínica dos participantes encontrase na Tabela 1.

Tabela 1- Estatística descritiva das variáveis sócio-demográficas e clínicas da amostra estudada. (Ribeirão Preto, 2003)

\begin{tabular}{|c|c|c|c|}
\hline Variável (n=97) & Intervalo & $\bar{x} *(s) * *$ & n (\%) \\
\hline Idade (anos) & $39-80$ & $60,1(9,64)$ & \\
\hline Tempo de cirurgia (anos) & $0,58-6,58$ & $2,56(1,5)$ & \\
\hline Número de enxertos coronarianos & $1-7$ & $2,88(1,26)$ & \\
\hline 1 a 2 & & & $44(45,4)$ \\
\hline 3 a 4 & & & $41(42,3)$ \\
\hline+4 & & & $12(12,4)$ \\
\hline \multicolumn{4}{|l|}{ Sexo } \\
\hline Feminino & & & $40(41,2)$ \\
\hline Masculino & & & $57(58,8)$ \\
\hline \multicolumn{4}{|l|}{ Estado Civil } \\
\hline Casado ou vivendo com alguém & & & $77(79,4)$ \\
\hline Viúvos & & & $11(11,3)$ \\
\hline Separados & & & $6(6,2)$ \\
\hline Solteiro & & & $3(3,1)$ \\
\hline \multicolumn{4}{|l|}{ Com quem mora } \\
\hline Com cônjuge e filhos & & & $52(53,6)$ \\
\hline Somente com o cônjuge & & & $25(25,8)$ \\
\hline Somente com o(s) filho(s) & & & $09(9,3)$ \\
\hline Sozinho & & & $07(7,2)$ \\
\hline Com outros familiares e/ou amigos & & & $04(4,1)$ \\
\hline \multicolumn{4}{|l|}{ Situação profissional/ ocupacional } \\
\hline Ativo & & & $18(18,6)$ \\
\hline Aposentado & & & $38(39,2)$ \\
\hline Aposentado com trabalho remunerado & & & $10(10,3)$ \\
\hline Desempregado, aguardando aposentadoria & & & $11(11,3)$ \\
\hline Dona de casa & & & $20(20,6)$ \\
\hline
\end{tabular}

* $\bar{x}$ (média) **s (desvio padrão)

Dos 97 participantes, $57(58,8 \%)$ eram do sexo masculino, $77(79,4 \%)$ eram casados e $86(88,7 \%)$ residiam com o cônjuge e/ou com os filhos. A idade variou entre 39 e 80 anos $(\bar{x}=60,1$ e $s=9,4)$ e 48 $(49,5 \%)$ pacientes estavam aposentados. Com relação à situação clínica, eles tinham um tempo médio de revascularização miocárdica de 2,56 anos e haviam recebido de 1 a $7(\bar{x}=2,8 ; s=1,26)$ enxertos coronarianos, sendo que $85(87,7 \%)$ receberam até 4 pontes.

Ao fazerem a avaliação subjetiva sobre a interferência da intervenção cirúrgica na qualidade de suas vidas, $69(71,1 \%)$ referiram melhora, $18(18,5 \%)$ referiram não terem observado diferença entre an- tes e depois da cirurgia e $10(10,4 \%)$ afirmaram piora na qualidade de vida.

Os resultados relacionados à nossa variável dependente, a auto-estima dos pacientes revascularizados, foram obtidos com a aplicação da Escala de Auto-Estima de Rosenberg ${ }^{(5)}$. A confiabilidade da escala, medida através da consistência interna de seus itens, foi verificada pelo alfa de Cronbach, que se mostrou adequado para a amostra estudada, com o valor de 0,796 . Com um intervalo possível de 10 a 40, foram obtidos escores entre 19 e 40 . A média obtida foi de $32,22 \pm 5,4$, o que indicou alta auto-estima entre os participantes.
A auto-estima de indivíduos que foram submetidos à cirurgia de revascularização do miocárdio 
Tatiana Victorelli P. Vargas Rosana Ap. S. Dantas Cristiane Franca L. Gois
Para testar a correlação entre a variável autoestima e outras variáveis contínuas utilizamos o teste de correlação de Pearson. Os valores obtidos mostraram que não havia correlações entre as variáveis auto-estima e idade $(\mathrm{r}=0,123 \mathrm{e}$ $\mathrm{p}=0,23)$ e auto-estima e tempo de revascularização $(r=0,086$ e $p=0,4)$.

Como gostaríamos de testar se a presença de um(a) companheiro(a) era importante para a autoestima, decidimos agrupar as quatro opções (solteiro, casado, viúvo, desquitado/divorciado) em apenas duas categorias: 1) estar casado(a) e ter alguém emocionalmente significante e 2) não estar casado e não ter alguém significante. Feito assim, obtivemos que $79,4 \%(n=77)$ dos entrevistados estavam na primeira categoria e $20,6 \%$ $(\mathrm{n}=20)$ na segunda. Também para testar se ter atividade profissional remunerada estava relacionada com a auto-estima, agrupamos as 5 opções (ativo, aposentado, aposentado com trabalho remunerado, desempregado e aguardando aposentadoria, dona de casa) em apenas duas: 1) ser profissionalmente ativo ou estar desenvolvendo atividades remuneradas após a aposentadoria e 2) não estar desenvolvendo atividades remuneradas. $\mathrm{O}$ resultado obtido foi $71,1 \%$ $(n=69)$ dos entrevistados não tinham renda proveniente de atividades profissionais e $28,9 \%$ $(\mathrm{n}=28)$ tinham essa renda.

As distribuições dos valores obtidos para a variável auto-estima com relação às variáveis: sexo, estar casado/ter alguém significante, ocupação remunerada, percepção dos participantes sobre a qualidade de suas vidas após a cirurgia de revascularização do miocárdio (piorou, melhorou e indiferente) e número de enxertos coronarianos recebidos (1 a 2, 3 a 4, ou mais do que 4 enxertos) estão apresentadas na Tabela 2.

Tabela 2 - Apresentação das médias $(\bar{x})$ e desvio-padrão $(s)$ de auto-estima em diferentes variáveis categóricas (sexo, atividade remunerada, estar casado, qualidade de vida e número de enxertos). (Ribeirão Preto, 2003)

\begin{tabular}{lll}
\hline Variáveis & $\mathbf{n}$ & $\overline{\boldsymbol{x}}(\mathbf{s})$ \\
\hline Sexo & & \\
$\quad$ Masculino & 57 & $33,27(5,14)$ \\
$\quad$ Feminino & 40 & $30,72(5,46)$ \\
Atividade remunerada & & \\
$\quad$ Sim & 28 & $33,91(5,13)$ \\
$\quad$ Não & 69 & $31,53(5,39)$ \\
Estar casado / Ter alguém significante & 77 & $32,14(5,39)$ \\
Sim & 20 & $32,50(5,54)$ \\
Não & & \\
QV*após a cirurgia & 69 & $32,53(5,27)$ \\
Melhorou & 10 & $29,20(5,84)$ \\
Piorou & 18 & $32,68(5,41)$ \\
Indiferente & & \\
Enxertos coronarianos & 44 & $32,11(5,71)$ \\
1 a 2 & & $32,50(5,00)$ \\
3 a 4 & 41 & $31,66(5,94)$ \\
mais de 4 & 12 & \\
\hline
\end{tabular}

* $\mathbf{Q V}=$ qualidade de vida

Para analisarmos se as diferenças encontradas nas categorias, eram estatisticamente significantes, utilizamos o teste não paramétrico de Mann-Whitney para sexo, atividade remunerada e estar casado/ter alguém significante e constatamos diferença apenas para a variável sexo $(\mathrm{p}=0,028)$. Assim, nossos resultados nos permitem dizer que a auto-estima entre os participantes do sexo masculino mostrou-se mais elevada do que entre os participantes do sexo feminino.

Para as variáveis com mais de 2 categorias usamos o teste de Kruskal Wallis. Embora, os participantes com maior auto-estima tivessem relatado uma melhora ou manutenção na quali- dade de suas vidas, após a intervenção cirúrgica, tal resultado não foi estatisticamente significante $(\mathrm{p}=0,2)$. O mesmo foi constatado com relação ao número de enxertos recebidos $(\mathrm{p}=0,9)$. Esses resultados podem indicar que os aspectos ligados ao tratamento cirúrgico, com a qualidade de vida percebida após a operação e o número de enxertos recebidos (refletindo a gravidade da doença) não parecem interferir na auto-estima dos pacientes.

\section{DISCUSSÃO}

Após concluirmos a análise dos dados obtidos, constatamos que a auto-estima dos parti- 
cipantes mostrou-se elevada. Esse resultado vai ao encontro daqueles obtidos por outros autores que utilizaram a mesma escala de auto-estima, em pacientes revascularizados e obtiveram valores entre 18 e $40(\bar{x}=33,4 ; s=4,9)^{(4)}$ e com pacientes após infarto agudo do miocárdio que apresentaram uma auto-estima entre 10 e 36 ( $\bar{x}=18,68$; $s=4,9)^{(11)}$. Comparativamente, os pacientes infartados apresentaram uma auto-estima menor do que os revascularizados, o que nos leva a pensar que o tratamento cirúrgico da DAC pode proporcionar aos pacientes a sensação de cura da doença, com conseqüente melhora da sensação de bemestar e de auto-estima.

A importância de se avaliar a auto-estima em pacientes cardíacos tem sido constatada em vários estudos. Presença de elevada auto-estima tem sido relacionada com baixos níveis de tensão emocional e depressão após revascularização do miocárdio ${ }^{(1)}$.

O conceito de auto-estima tem sido usado como sinônimo de auto-eficácia. A relação entre esses dois conceitos ainda é controversa e pesquisadores têm apresentado que a escala de auto-estima de Rosenberg tem forte correlação com medidas de auto-eficácia, ou seja, ela também indicaria o sentimento de confiança que o indivíduo tem de realizar tarefas propostas a ele ${ }^{(6)}$. Em se tratando do paciente revascularizado isso é de suma importância uma vez que, após a cirurgia, ele necessitará desempenhar novas e complexas tarefas para manter a sua saúde como, por exemplo: uso de múltiplas drogas, controle da dieta, realização de atividades físicas.

Comparando nossos resultados com os obtidos em uma amostra de revascularizados americanos $^{(4)}$, pudemos observar que os dados obtidos em algumas das variáveis estudadas foram semelhantes como, por exemplo: maior parte da amostra ser do sexo masculino, as médias de idade dos grupos e estado civil dos pacientes estudados. Mas também existiram dados divergentes, como o tempo de cirurgia que foi maior no nosso estudo. Embora vivendo em países distintos e com algumas características clínicas diferentes, podemos afirmar que essas diferenças não interferiram na auto-estima de ambas as amostras, ou seja, tanto os revascularizados brasileiros quanto os norte-americanos apresentaram elevada auto-estima.

Com relação à confiabilidade do instrumento usado para medir a variável, auto-estima, tivemos um alfa de 0,79 , valor esse consistente com aqueles valores publicados em outros estudos que usaram a escala de Rosenberg ${ }^{(4,10)}$.
Em nosso estudo, constatamos que a auto-estima esteve correlacionada com o sexo dos participantes, sendo as mulheres aquelas que referiram menores valores para a auto-estima. Embora tal resultado já tivesse sido constatado anteriormente ${ }^{(8)}$, ele nem sempre tem sido estatisticamente significante $^{(11)}$. Acreditamos que as mulheres, principalmente aquelas com baixa escolaridade e idosas, ainda trazem as consequências de uma educação voltada para o papel de mãe e esposa, vivendo em função dos pais, maridos e filhos. Assim, ao avaliarem a si próprias, através da escala de Rosenberg, apresentam pontuações menores que os pacientes do sexo masculino.

Os resultados referentes à relação entre autoestima e idade têm sido controversos. Apesar da nossa amostra ser heterogênea com relação a variável idade, com participantes jovens e idosos (variando entre 39 e 80 anos), não constatamos uma correlação entre idade e auto-estima, como em outro estudo com pacientes cardíacos ${ }^{(11)}$. No entanto, outras pesquisas têm constatado que a autoestima esteve negativamente correlacionada com a idade ${ }^{(9)}$ ou que a mesma aumenta com o envelhecimento, tendo os idosos maior auto-estima ${ }^{(8)}$.

Estudo feito com o objetivo de analisar a influência da idade sobre a auto-estima, também discute que a classe social e o nível educacional têm sido considerados como aspectos que influenciam a auto-estima ${ }^{(12-13)}$. Os autores referem que uma maior escolaridade pode auxiliar as pessoas a se mobilizarem para vencer obstáculos relacionados aos problemas de saúde e suas incapacidades e resgatar o controle sobre suas vidas ${ }^{(8)}$. Embora em nosso estudo não tenhamos levantado o grau de escolaridade e a renda familiar dos participantes, como variáveis caracterizadoras da condição social, estudos realizados na mesma instituição, abordando outras amostras da população de coronariopatas atendidos no ambulatório de Cardiologia, nos apresentaram que os pacientes possuem baixo grau de escolaridade (menos que 8 anos de instrução) e renda familiar menor do que seis salários mínimos ${ }^{(14-15)}$. Diante disso, o resultado obtido, ou seja, elevada auto-estima entre os pacientes revascularizados atendidos nessa instituição hospitalar, foi um aspecto inesperado em nosso estudo. Ao compararmos esse resultado com aqueles obtidos em estudos usando a mesma escala de auto-estima, com populações de maior escolaridade e renda ${ }^{(4,11)}$ constatamos valores semelhantes. Essa constatação nos faz questionar afirmações anteriores sobre a correlação negativa existente entre auto-estima e classe social ou auto-estima e nível educacional trazidas pela literatura ${ }^{(12-13)}$.
A auto-estima de indivíduos que

foram submetidos à cirurgia de revascularização do miocárdio

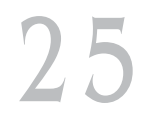

Rev Esc Enferm USP 2005; 39(1):20-7. 
Tatiana Victorelli P. Vargas Rosana Ap. S. Dantas Cristiane Franca L. Gois
Ao levarmos em conta que, em nossa amostra, 71,1\% $(\mathrm{n}=69)$ dos participantes não possuíam renda proveniente de atividades profissionais, como fonte de complementação da renda familiar, e que muitos deles viviam exclusivamente da aposentadoria, esperávamos uma relação entre as variáveis auto-estima e desempenho de atividades remuneradas, a qual não foi encontrada.

Nesse estudo, estar casado ou ter alguém emocionalmente significante, foi considerado um indicador de suporte social para os pacientes. Estado civil é um indicador de apoio social, instrumental e emocional ${ }^{(16)}$. No entanto, apesar da maioria dos participantes $(79,4 \%)$ serem casados ou terem alguém emocionalmente significante, não houve uma correlação estatística entre auto-estima e estado civil, o que nos faz questionar até que ponto estar casado ou ter alguém emocionalmente significante poderia influenciar a autoestima dos pacientes estudados.

Não encontramos diferenças na percepção de como a cirurgia afetou a qualidade de vida dos pacientes (piorou, melhorou, foi indiferente). Em estudos anteriores foi constatada uma relação positiva entre a auto-estima e a qualidade de vida, apresentando uma relação positiva entre as mes$\operatorname{mas}^{(3,4)}$. Outros autores têm salientado que altos níveis de auto-estima estão provavelmente relacionados com a percepção de um estado de saúde entre os pacientes excelente ou ótimo ${ }^{(8,17)}$. O estado de saúde percebido pelo indivíduo tem sido relacionado, forte e positivamente, com o controle da própria saúde, a auto-eficácia e a autoestima, sendo que a incapacidade física também tem um forte efeito negativo sobre o controle da saúde e da auto-estima e fraco efeito negativo sobre auto-eficácia.

\section{CONCLUSÃO E IMPLICAÇÕES PARA A PRÁTICA DE ENFERMAGEM}

Foi constatada elevada auto-estima entre os pacientes revascularizados na amostra estudada. A correlação entre auto-estima e as variáveis sócio-demográficas foi estabelecida apenas para o sexo, tendo os homens uma auto-estima mais elevada do que as mulheres. Esse resultado é importante para o enfermeiro no planejamento da reabilitação dos revascularizados, pois indica que o mesmo deverá desenvolver estratégias diferentes para as pacientes do sexo feminino. Por exemplo, intervenções que enfatizem a auto-estima dessas mulheres irão contribuir para facilitar as mudanças de comportamentos tão necessárias no processo de reabilitação cardíaca. O tempo de cirurgia e o número de enxertos não influenciaram a auto-estima dos participantes, assim como a avaliação do procedimento cirúrgico sobre a qualidade de suas vidas.

Acreditamos que os enfermeiros, entre outros profissionais de saúde que cuidam de indivíduos após revascularização do miocárdio, devemos estar atentos para as características psicossociais desses pacientes, entre elas, a autoestima. A avaliação da auto-estima poderá contribuir na detecção daqueles indivíduos que terão maiores dificuldades para se reabilitarem, uma vez que baixa auto-estima pode refletir em sentimentos de incapacidade para mudar e manter comportamentos favoráveis à saúde cardiovascular, tais como: praticar exercícios físicos e deixar de fumar. Outro aspecto importante é que a presença de elevada auto-estima tem sido associada a baixos níveis de depressão e tensão após cirurgia de revascularização do miocárdio.

\section{REFERÊNCIAS}

(1) Logsdon MC, Usui, WM, Cronin SN, Miracle VA. Social support and adjustment in women following coronary artery bypass surgery. Health Care Women Int 1998; 19(1): 61-70.

(2) Artinian NT, Duggan C, Miller P. Age differences in patient recovery patterns following coronary artery bypass surgery. Am J Crit Care 1993; 2(6):453-61.

(3) Motzer SU, Stewart BJ. Sense of coherence as a predictor of quality of life in persons with coronary heart disease surviving cardiac arrest. Res Nurs Health 1996; 19(4):287-98.
(4) Dantas RA, Motzer SA, Ciol, MA. The relationship between quality of life, sense of coherence, and self-esteem in persons after coronary artery bypass graft surgery. Int J Nurs Stud 2002; 39(7):745-55.

(5) Rosenberg M. Society and the adolescent selfimage. New Jersey: Princeton University Press; 1965.

(6) Stanley KD, Murphy MR. A comparison of general self-efficacy with self- esteem. Genet Soc Gen Psychol Monogr 1997; 123(1):81-99. 
(7) Rossi SR, Rossi JS. Concepts and theoretical models. In: Iairath N. Coronary heart disease \& risk factor management. A nursing perspective. Philadelphia: W.B. Saunders; 1999. p. 47-69.

(8) Schieman S, Campbell JE. Age variations in personal agency and self-esteem: the context of physical disability. J Aging Health 2001; 13(2):155-85.

(9) Lee G, Shehan CL. Social relations and the selfesteem of older persons. Res Aging 1989; 11(4):427-42.

(10) Dini GM. Adaptação cultural, validade e reprodutibilidade da versão brasileira da escala de auto-estima de Rosenberg. [dissertação]. São Paulo (SP): Universidade Federal de São Paulo; 2001.

(11) Conn VS, Taylor SG, Abele PB. Myocardial infarction survivors: age and gender differences in physical health, psychosocial state and regimen adherence. J Adv Nurs 1991; 16(9):1026-34.

(12) Ross C, Mirowsky J. Explaining the social patterns of depression: control and problem solving - or support and talking? J Health Soc Behav 1989; 30(2):206-19.
(13) Turner RJ, Roszell P. Personal resources and the stress process. In: Avison WR, Gotlib, IH, editors. Stress and mental health: contemporary issues and prospects for the future. New York: Plenum; 1994.

(14) Dantas RAS, Aguillar OM. Perfil de pacientes com infarto agudo do miocárdio na perspectiva do modelo de "Campo de saúde". Rev Bras Enferm 1998; 51(4):571-88.

(15) Dantas RAS, Aguillar OM, Barbeira CBS. Retorno às atividades ocupacionais e sexuais após a cirurgia de revascularização do miocárdio. Rev Lat Am Enferm 2001; 9(4):26-31

(16) Allen SM, Ciambrone D, Welch LC. Stage of life and social support as a mediator of mood state among persons with disability. J Aging Health 2000; 12(3):318-41.

(17) Cott CA, Gignac MA, Badley EM. Determinants of self rated health for Canadians with chronic disease and disability. J Epidemiol Community Health 1999; 53(11):731-6.
A auto-estima de indivíduos que foram submetidos à cirurgia de revascularização do miocárdio 